\title{
Evaluation of protective effect of DNA vaccination with genes encoding antigens GRA4 and SAG1 associated with GM-CSF plasmid, against acute, chronical and congenital toxoplasmosis in mice
}

\author{
Marie-Noëlle Mévélec ${ }^{\mathrm{a}, *}$, Daniel Bout ${ }^{\mathrm{a}}$, Benoît Desolme ${ }^{\mathrm{a}}$, Hervé Marchand ${ }^{\mathrm{a}}$, Rémy Magnéa ${ }^{\mathrm{a}}$, \\ Odile Bruneel $^{\mathrm{a}}$, Dominique Buzoni-Gatel ${ }^{\mathrm{b}}$ \\ ${ }^{a}$ Université François-Rabelais de Tours, INRA, UMR 483 Université-INRA d'Immunologie Parasitaire et Vaccinologie, \\ IFR Agents transmissibles et Infectiologie, UFR des Sciences Pharmaceutiques, 31 Avenue Monge, 37200 Tours, France \\ b Institut Pasteur, Unité Réponse précoce aux Parasites et Immunopathologie, Paris, France
}

\begin{abstract}
To develop a multiantigenic vaccine against toxoplasmosis, two Toxoplasma gondii antigens, SAG1 and GRA4 selected on the basis of previous immunological and immunization studies, were chosen. We showed that DNA-based immunization with plasmids expressing GRA4 (pGRA4) or SAG1 (pSAG1mut) reduced mortality of susceptible C57BL/6 mice upon oral challenge with cysts of the 76K type II strain (62\% survival). Immunization with pGRA4 and pSAG1mut, enhanced the protection (75\% survival). This protection was further increased by co-inoculation with a plasmid encoding the granulocyte-macrophage colony-stimulating factor (GM-CSF) (87\% survival). This latter DNA cocktail provided significant protection of less susceptible outbred Swiss OF1 mice against the development of cerebral cysts. A significantly higher survival of newborns from immunized outbred mice exposed to infection during gestation was observed $(4.25 \pm 3.77$ live pups/litter) in comparison to non-immunized mice (1.08 \pm 2.15 live pups/litter) without preventing parasite vertical transmission. Analysis of the immune response showed that protected animals developed a specific humoral and cellular Th1 response to native T. gondii SAG1 and GRA4 antigens. Our data demonstrated that protection was improved by associating antigens (SAG1 and GRA4) and cytokine (GM-CSF) for further development of a multiantigenic vaccine against toxoplasmosis.
\end{abstract}

Keywords: Toxoplasma gondii; DNA vaccine; SAG1; GRA4

\section{Introduction}

Toxoplasma gondii is an obligate intracellular parasite responsible for toxoplasmosis in humans and other warmblooded animals. Although generally mild in its effects on immunocompetent humans, toxoplasmosis can cause severe damage in cases of congenital transmission. In an immunocompromised host, the recrudescence of latent infection can lead to severe or lethal damage. Toxoplasmosis also causes abortion and loss of offspring in animals, es-

\footnotetext{
* Corresponding author. Tel.: +332 47 367185/86; fax: +33 247367252 .

E-mail address: mevelec@univ-tours.fr (M.-N. Mévélec).
}

pecially in sheep and goats. The effective commercial live vaccine for sheep is not of practical use and is not appropriate for human use, as it might revert to a pathogenic strain [1].

Several vaccination trials against toxoplasmosis have already been performed. Among the well-defined antigen candidate vaccines, SAG1, the $30-\mathrm{kDa}$ surface antigen of the proliferative form of $T$. gondii, is the most studied. Results obtained in animal models using purified native SAG1 $[2,3]$ subcutaneously or intranasally administered, synthetic peptides derived from SAG1 [4] or recombinant SAG1 (rSAG1) produced in Escherichia coli [5,6] have been encouraging but showed only partial protection. The combination of recombi- 
nant IL-12 with rSAG1 was shown to redirect the unprotective Th2-type immune response elicited by rSAG1 alone towards a Th1-type immune response that partially protects CBA/J mice against oral infection by $T$. gondii [5]. The effect of vaccination with SAG1 was also evaluated against congenital toxoplasmosis in BALB/c and CBA/J mice [7]. Vaccination of $\mathrm{BALB} / \mathrm{c}$ mice with a recombinant SAG1 reduced the number of infected fetuses by $50 \%$ and was associated with a mixed type 1 and 2 immunity, whereas vaccination of CBA/J mice increased the number of infected fetuses by $50 \%$ and was associated with a predominant type- 2 response.

Intramuscular DNA vaccination that readily elicited a Th1-type response [8] is an attractive vaccine approach against $T$. gondii. Recently we and others have reported a strategy based on plasmid DNA encoding $T$. gondii antigens, suggesting that nucleic acid vaccination can provide protection against $T$. gondii infection in mice. $\mathrm{C} 3 \mathrm{H}$ and $\mathrm{BALB} / \mathrm{c}$ mice immunized with a plasmid expressing SAG1 and challenged by intraperitoneal injection with a lethal dose of tachyzoïtes from the RH strain were significantly protected [9]. C57BL/6 mice immunized with a plasmid expressing SAG1 associated with a plasmid expressing GM-CSF and orally challenged with cysts of the ME49 strain of $T$. gondii were $100 \%$ protected whereas no protection was observed following challenge by intraperitoneal injection of tachyzoïtes from the RH strain [10]. Vaccination of BALB/c mice with a plasmid expressing SAG1 and orally challenged with the Beverly type II strain afforded protection against adult acquired infection but did not prevent maternofoetal transmission in previously vaccinated dams infected during pregnancy [11].

We have demonstrated that intramuscular immunization of C57BL/6 mice with a plasmid expressing GRA4, a dense granule antigen of $T$. gondii provides significant protection against a lethal oral infection with $T$. gondii cysts (62\% survival). Futhermore immune responses and protection were improved by co-inoculation of a plasmid expressing GMCSF (75\%) [12].

Accumulating evidence indicates that multi-antigen immunizations are needed for the development of an effective vaccine against complex pathogens such as parasites [13].

In the present study, mice were immunized with the plasmids expressing GRA4 and SAG1 antigens that can elicit potentially protective immune responses, SAG1 being expressed only by tachyzoïtes, GRA4 being expressed by both tachyzoïtes and bradyzoïtes. We used one strain of inbred mice highly susceptible to T. gondii: C57BL/6. Outbred OF1 mice, intermedialty resistant, were used to circumvent genetic restriction of protection. Protection was evaluated against both chronic infection and congenital toxoplasmosis. The antibody and lymphoproliferative responses to SAG1 and GRA4 and as well as the cytokine patterns of in vitro restimulated splenocytes were also evaluated.

\section{Materials and methods}

\subsection{Mice}

Seven- to eight-week-old female inbred mice (CBA/J, C57BL/6) and female outbred mice (Swiss OF1), were purchased from Janvier, France.

\subsection{Parasites}

Tachyzoites of the RH strain of T. gondii were harvested from the peritoneal fluid of Swiss OF1 mice that had been intraperitoneally infected 3-4 days earlier. The $76 \mathrm{~K}$ strain of T. gondii [14] was maintained in the laboratory by passage of infective brain homogenate in CBA/J mice, and used for all experimental infections. The $76 \mathrm{~K}$ strain is a type II isolate [15]. Type II isolates are predominant in human congenital toxoplasmosis [16].

\subsection{Bacterial strains and growth conditions}

E. coli $\mathrm{DH} 5 \alpha$ was the host for DNA manipulation experiments. Bacteria were propagated in Luria Bertani broth or on Luria Bertani agar supplemented with ampicillin $(100 \mu \mathrm{g} / \mathrm{ml})$ where appropriate.

\subsection{Expression vectors}

The SAG1 DNA with a signal sequence at the $\mathrm{N}$ terminal and a predicted glycolipid anchor at the C-terminal [17] was amplified by PCR from genomic DNA of $T$. gondii tachyzoites (RH strain). Primers (Génome Express, France) used to amplify SAG1 sequence were $5^{\prime}$ CCACTTCATGAATTCTTCTGGTTTTTT 3' (forward) and 5' GAGACTCGAGCACAACGGTGATCACTCA $3^{\prime}$ (reverse), introducing respectively the EcoRI and the XhoI restriction sites for ligation into EcoRI- and XhoI-cut expression vector pcDNA3 (Invitrogen) to produce pSAG1. This eukaryotic expression vector contains the cytomegalovirus early promotor/enhancer sequence and the polyadenylation and $3^{\prime}$ splicing signals from bovine growth hormone. The asparagine codon (AAC) of the N-linked glycosylation site $\mathrm{Asn}_{241}$-AlaSer [14] was substituted for Gln codon (CAG) by point mutations using QuickChange ${ }^{\mathrm{TM}}$ Site-Directed Mutagenesis kit (Stratagene) with the primers 5' GGCAGGGTCAGGCTTCGAGTGATAAGG $3^{\prime}$ (forward) and 5' CCTTATCACTCGAAGCCTGACCCTGCC $3^{\prime}$ (reverse) which introduce specific mutations. The resulting plasmid was termed pSAG1mut. The plasmid encoding GRA4 (pGRA4) has been previously described [12]. The plasmid encoding murine granulocyte-macrophage colony-stimulating factor (pGMCSF) was kindly provided by Dr. H.C.J. Ertl (Wistar Institute, Philadelphia, PA). Plasmids were purified from transformed E. coli $\mathrm{DH} 5 \alpha$ by anion exchange chromatography (Endofree plasmid giga kit, Qiagen $\mathrm{GmBH}$ ), dissolved in sterile endotoxin-free phosphate-buffered saline (Sigma) and 
stored at $-20^{\circ} \mathrm{C}$. The integrity of the DNA plasmids was checked by agarose gel electrophoresis after digestion with appropriate restriction enzymes or without digestion (supercoiled form was predominant). DNA concentration was determined by absorbance at $260 \mathrm{~nm}$. The OD 260/280 ratios for purified DNA were 1.80-1.95, indicating that preparations were free of major protein contamination.

\subsection{In vitro expression of $S A G 1$}

COS-7 cells were transfected with either pSAG1, pSAG1mut or a control plasmid pcDNA3, using a polycationic liposome reagent (LipofectAMINE ${ }^{\mathrm{TM}}$, GIBCO BRL) as previously described [12].

For immunoblots with transfected cells and purified $T$. gondii tachyzoites (RH strain), sodium dodecyl sulfatepolyacrylamide gel electrophoresis and immunoblotting were performed as previously described [18]. Cells $\left(6 \times 10^{6}\right)$ were harvested $48 \mathrm{~h}$ after transfection, and suspended in $150 \mu \mathrm{l}$ sodium dodecyl sulfate polyacrylamide electrophoresis sample buffer, sonicated and heated at $100{ }^{\circ} \mathrm{C}$ for $3 \mathrm{~min}$. The separated proteins were probed with anti-SAG1 Mab 1E5 [19], a T. gondii immune mouse serum. Bound antibodies were detected with alkaline phosphatase-conjugated goat anti-mouse IgG (Sigma). Molecular mass standards (Prestained SDS-PAGE standards, Low range, Bio-Rad) were used.

\subsection{Intramuscular immunization}

Mice were immunized as previously described [12]. Prior to immunization, each mouse was injected with $100 \mu \mathrm{l}$ of $10 \mu \mathrm{M}$ cardiotoxin (Latoxan, Rosans, France) into the tibialis anterior muscles of both hind legs to enhance plasmid DNA uptake [20]. Five days later, the regenerating mouse muscles of both hind legs were then injected with $50 \mu \mathrm{g}$ pSAG1mut or $50 \mu \mathrm{g}$ pGRA 4 or $25 \mu \mathrm{g}$ pSAG1mut plus $25 \mu \mathrm{g}$ pGRA4 or $25 \mu \mathrm{g}$ pSAG1mut plus $25 \mu \mathrm{g}$ pGRA4 with $25 \mu \mathrm{g}$ of pGMCSF in $50 \mu \mathrm{l}$ PBS, using syringes with 301/2-gauge needles (Microlance 3, Becton Dickinson). The mice were boosted in the same way on day 14 and with plasmids encoding antigens without pGM-CSF on day 28. Control groups included mice receiving empty pcDNA3 or mice left untreated (no cardiotoxin, no plasmid).

\subsection{Evaluation of protection against acute and chronic toxoplasmosis}

C57BL/6 mice, were orally infected with 40 cysts of the $76 \mathrm{~K}$ strain obtained from brains of chronically infected mice (lethal dose). Survival was recorded for 30 days. Protection against the chronic phase of infection was investigated in outbred Swiss OF1 mice. Brain cysts were enumerated 1 month after challenge with 70 cysts of the $76 \mathrm{~K}$ strain. The mouse brains were homogenized with a mortar and pestle in $5 \mathrm{ml}$ of PBS. The cysts were counted microscopically in eight samples $(10 \mu \mathrm{l}$ each $)$ of each brain homogenate.

\subsection{Evaluation of protection against congenital toxoplasmosis}

Mice were immunized with $25 \mu \mathrm{g}$ pSAG1mut plus $25 \mu \mathrm{g}$ pGRA4 with $25 \mu \mathrm{g}$ of pGM-CSF as described above. Fifteeen days after the third immunization, groups of two virgin Swiss OF1 mice were housed with one male for 4 days. Then the females, housed separately to rear their pups, were orally infected between the 7th and 10th days of pregnancy with 70 cysts of the $76 \mathrm{~K}$ strain. All the dams were allowed to nurture their offspring normally, no cross-fostering procedure was used. The number of live pups were recorded for 30 days. Pups were weighed 8, 15 and 30 days after birth. The pups were sacrificed 30 days after birth and their brain cysts were counted microscopically in eight samples (10 $\mu$ l each) of each brain homogenate $(5 \mathrm{ml}$ of PBS). Two control groups were composed of mice left untreated (no cardiotoxin, no plasmid) and infected between the 7th and 10th days of pregnancy or left untreated and not infected during pregnancy.

\subsection{Assessment of the humoral response to DNA immunization}

Two weeks after the last immunization, sera were tested for the presence of IgG antibodies against $T$. gondii antigens by ELISA, using a GST recombinant GRA4 protein (NC) or the SAG1 protein purified by immunoaffinity, as previously described [3,21]. Anti-T. gondii IgG subclasses analysis was performed by ELISA using a toxoplasma sonicate (TAg) prepared as previously described [22] and the alkaline phosphatase-conjugated anti-mouse IgG1 and IgG2a at 1:1000 (Cappel, West Chester, PA). Optimal dilution of each serum was determined after titration analysis.

\subsection{T. gondii-specific proliferative response}

Three mice from each group were killed 6 days after the last immunization and their spleens were removed and pressed. Single cell suspensions were obtained by filtration through nylon mesh. The erythrocytes in the spleen cell suspension were removed by lysis (hypotonic shock) and the remaining cells were washed and suspended in RPMI 1640 medium (GIBCO) supplemented with 5\% fetal calf serum, HEPES ( $N$-2-hydroxyethylpiperazine- $N N^{\prime}-2$ ethanesulfonic acid) (10 mM), L-glutamine ( $2 \mathrm{mM})$, sodium pyruvate $(1 \mathrm{mM}), \beta$-mercaptoethanol $(50 \mu \mathrm{M})$, penicillin $(100 \mathrm{U} / \mathrm{ml})$, streptomycin $(100 \mu \mathrm{g} / \mathrm{ml})$. They were seeded in triplicate in flat-bottomed 96-well microtiter plates (Costar) at $3 \times 10^{5}$ cells per well in $200 \mu$ l culture medium alone or with various concentrations of TAg or $4 \mu \mathrm{g} / \mathrm{ml}$ concanavalin A. Toxoplasma sonicate (TAg) was prepared as previously described [22]. The plates were incubated for $84 \mathrm{~h}$ in $5 \% \mathrm{CO}_{2}$ at $37^{\circ} \mathrm{C}$ and pulsed with $37 \mathrm{KBq}\left[{ }^{3} \mathrm{H}\right]$ thymidine per well for the 
final $18 \mathrm{~h}$. The cells were ruptured by freezing and thawing and cell debris were collected on glass fiber filters. The incorporated radioactivity was quantified by liquid scintillation counting. Proliferation was expressed as the stimulation index (counts per minute for stimulated cells/counts per minute for unstimulated cells).

\subsection{Cytokine quantification}

Spleen cell proliferation was assayed as described above. Cell-free supernatants were harvested and assayed for interleukin-4 (IL-4) activity at $24 \mathrm{~h}$, for interleukin-10 (IL10) activity at $72 \mathrm{~h}$, and for gamma-interferon (IFN- $\gamma$ ) activity at $96 \mathrm{~h}$. The IL-4, IL-10 and IFN- $\gamma$ concentrations were evaluated using a commercial ELISA kit according to the manufacturer's instructions (DuoSet, Genzyme). Cytokine concentrations were determined by reference to standard curves constructed with known amounts of mouse recombinant IFN- $\gamma$, IL-10 or IL-4. The sensitivity limits for the assays were $20 \mathrm{pg} / \mathrm{ml}$ for IFN- $\gamma, 50 \mathrm{pg} / \mathrm{ml}$ for IL- $10,10 \mathrm{pg} / \mathrm{ml}$ for IL-4.

\subsection{Statistical analysis}

The levels of significance of the differences between the groups of mice were determined using Wilcoxon's signedrank test for mortality, the Kruskall-Wallis and MannWhitney tests for viability of pups and using Student's test for the other analyses.

\section{Results}

\subsection{Expression of SAG1}

SAG1 was synthetized in a eukaryotic system. For that COS-7 cells were transfected with SAG1-encoding plasmid mutated (pSAG1mut) or not (pSAG1) on N-linked glycosylation site, or with empty pcDNA3, for $48 \mathrm{~h}$. The protein extracts were then analyzed by SDS-PAGE and western blotting (Fig. 1). A band at about $30 \mathrm{kDa}$ was recognized by antiSAG1 Mab 1E5 in protein extracts of cells transfected with pSAG1mut (Fig. 1, lane 2A) similar with that observed in protein extracts of $T$. gondii tachyzoites (Fig. 1, lane 1A). This band was also detected with sera collected from $T$. gondii infected mice (Fig. 1, lane 2B). SAG1 protein was not detected in those cells transfected with empty pcDNA3 (Fig. 1, lanes $4 \mathrm{~A}$ and $\mathrm{B}$ ). Therefore, pSAG1mut appeared to direct the synthesis of an antigenic SAG1 protein by eukaryotic cells cultured in vitro. In the extracts of cells transfected with pSAG1, the synthesized protein migrated as a band 4-5 kDa larger that the natural protein on SDS-polyacrylamide gel (Fig. 1, lanes 3A and B). This suggested that N-linked glycostylation site is being used in COS-7 cells, which was confirmed by tunicamycin treatment of COS-7/pSAG1 cultures (data not shown).

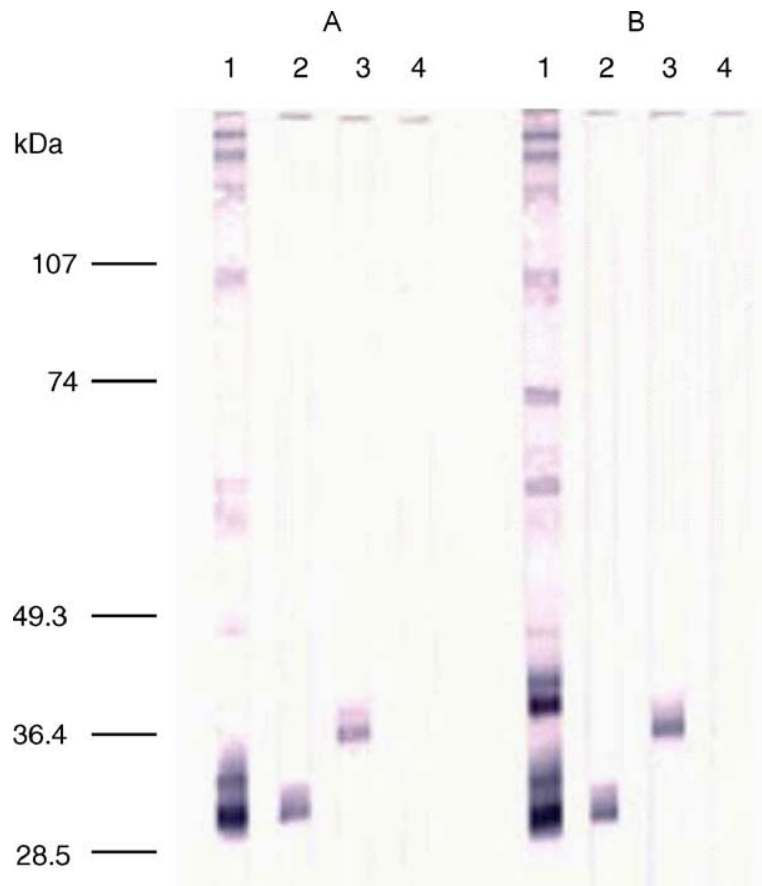

20.9

Fig. 1. Immunoblot analysis of expression and antigenicity of $\mathrm{N}$ glycosylation site mutated SAG1 protein. Lysates of COS-7 cells transfected with pSAG1mut (lane 2), pSAG1 (lane 3) or empty pcDNA3 (lane 4), and lysate of $T$. gondii tachyzoites (lane 1), were electrophoresed in a $12 \%$ SDS-polyacrylamide gel, transferred to nitrocellulose and reacted with an anti-SAG1 MoAb (A) or serum from a T. gondii infected mouse (B). Bound antibodies were detected using alkaline phosphatase-conjugated goat antimouse IgG. Positions of molecular size standards are indicated on the left of the panel.

The immunization experiments were pursued using pSAG1mut that is more suitable for the production of a native like form of SAG1 as compared to pSAG1, even if the SAG1 $\mathrm{N}$-glycosylation failed to affect its antigenicity.

\subsection{Protection against acute and chronic infection}

To determine whether it is possible to induce protection against mortality, groups of C57BL/6 mice were immunized with pSAG1mut alone, or pSAG1mut plus pGRA4, or a mixture of pSAG1mut, pGRA4 plus plasmid encoding GM-CSF (pGM-CSF) and challenged. Mice immunized with pcDNA3 or untreated mice served as negative controls.

Two weeks after the last immunization, mice were orally challenged with a lethal dose of cysts. Mortality was recorded 


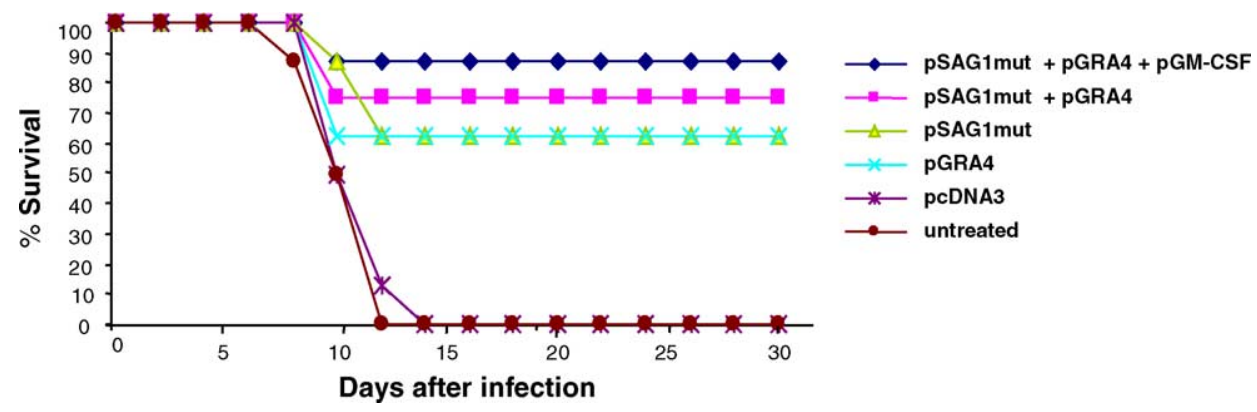

Fig. 2. Protection of C57BL/6 mice against Toxoplasma infection. The mice ( $n=8 /$ group) were immunized on days 0 and 14 with $100 \mu \mathrm{g}$ pAG1mut or $100 \mu \mathrm{g}$ pGRA4 or $50 \mu \mathrm{g}$ pSAG1mut plus $50 \mu \mathrm{g}$ pGRA4 or $50 \mu \mathrm{g}$ pSAG1mut plus $50 \mu \mathrm{g}$ pGRA4 with $50 \mu \mathrm{g}$ pGM-CSF. The mice were immunized on day 28 with plasmids encoding antigens without pGM-CSF. Two groups of mice, untreated and empty pcDNA3 inoculated were included as controls. The mice were challenged by gavage with 40 cysts of $76 \mathrm{~K}$ strain, 2 weeks after the last immunization, and observed daily for mortality. These data are representative of two independent experiments. $P<0.0003$, pSAG1mut vs. pcDNA3; $P<0.0007$, pGRA4 vs. pcDNA3; $P<0.0001$, pSAG1mut + pGRA4 vs. pGRA4; $P=0.01$, pSAG1mut + pGRA4 vs. pSAG1mut; $P=0.004$, pSAG1mut + pGRA4 + pGM-CSF vs. pSAG1mut + pGRA4.

daily for 1 month (Fig. 2). Immunization of C57BL/6 mice with pSAG1mut significantly increased the survival rate of these mice $(62 \%$ survival was recorded $[n=8])(P<0.0003)$ in comparison with control mice. Similar results were obtained with pGRA4-immunized mice (62\% survival was recorded $[n=8])(P<0.0007)$. A significant increase in the survival rate $(75 \%$ survival $[n=8])$ was observed in the mice injected with pSAG1mut plus pGRA4 in comparison with mice immunized with pGRA4 $(P<0.0001)$ or pSAG1mut $(P=0.01)$. The protection was further increased in the mice injected with pSAG1mut, pGRA4 plus pGM-CSF (87\% survival $[n=8])$ in comparison with mice immunized with pSAG1mut plus pGRA4 $(P=0.004)$. None of the untreated $(n=8)$ or pcDNA3 $(n=8)$ injected control groups survived infection.

Protection against the chronic phase of infection was investigated in outbred Swiss OF1 mice. Mice were immunized with a mixture of pSAG1mut, pGRA4 plus pGM-CSF. Mice immunized with pcDNA3 served as negative controls. Mice were orally infected with a nonlethal dose 2 weeks following the last immunization. There was a significant decrease in the number of tissue cysts from the vaccinated group compared with the control group $(P=0.0014)$ (Fig. 3).

\subsection{Protection against congenital toxoplasmosis}

Preliminary experiments demonstrated that survival of offspring from mice orally infected with 70 cysts of the $76 \mathrm{~K}$ strain between 7 th and 10th days of pregnancy was reduced (cumulative mortality of $66 \%$ at 30 days after birth). In addition, surviving pups displayed body weights strongly reduced by $50 \%$ as compared to those from uninfected mice 15 days after birth. The infection status of the offspring determined by brain cyst load at 30 days after birth, showed that all stillborn pups are infected. In this model, the protective effect of a chronical infection against congenital toxoplasmosis was assessed. Offspring from mice orally infected 1 month before mating with cysts of the $76 \mathrm{~K}$ strain and reinfected during pregnancy survived (100\%) and the litter size was similar in chronically infected mice $(12.33 \pm 1.15)$ and in uninfected mice $(12.30 \pm 1.96)$. Offspring from mice orally infected 1 month before mating and reinfected during pregnancy did not weight less than those from uninfected mice $(8.04 \pm 0.76 \mathrm{~g}$ versus $8.89 \pm 0.65 \mathrm{~g}, 15$ days after birth). Brain cysts were detected 30 days after birth in all pups born from infected mice $(612 \pm 263$ per brain, $n=10)$. One pup born from mice orally infected 1 month before mating and reinfected during pregnancy exhibited 63 brain cysts (90\% cyst reduction). In our experimental conditions, no cysts were detected in $96.6 \%$ of other pups ( $n=29)$ (>90\% cyst reduction). It is to note that no cross-fostering procedure was used to prevent the possible infection via lactation [23,24]. However, in such conditions we are able to demonstrate that $\mathrm{OF} 1$ dams infected several weeks before mating and reinfected during pregnancy develop immunity capable of protecting their pups compared to dams orally infected during pregnancy. Therefore, the protective effect of pSAG1mut, pGRA4 plus pGM-CSF was assessed in this animal model. A significantly greater survival rate in litters from mice immunized with a mixture of pSAG1mut,

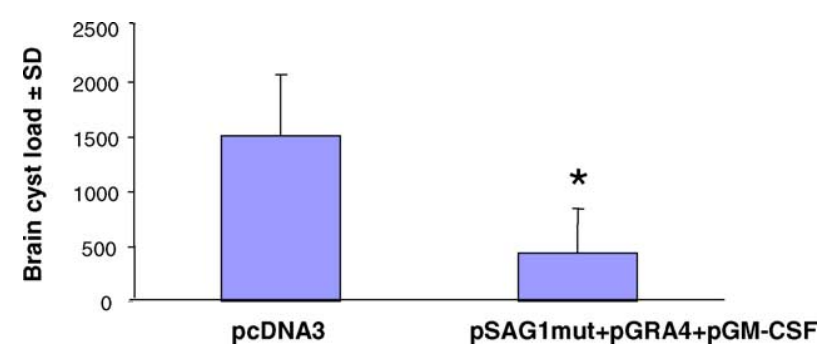

Fig. 3. Brain cyst load of outbred Swiss OF1 mice immunized with a mixture of pSAG1mut plus pGRA4 with pGM-CSF. Mice were immunized on days 0 and 14 with pSAG1mut, pGRA4 and pGM-CSF (50 $\mu \mathrm{g}$ each) and were immunized on day 28 with plasmids encoding antigens without pGMCSF. Mice inoculated with empty pcDNA3 were included as controls. Two weeks after the last immunization, the mice were orally infected with 70 cysts of $76 \mathrm{~K}$ strain. Brain cyst load was evaluated 1 month after challenge. Results from one of three similar experiments are shown. Vaccination with pSAG1mut plus pGRA4 with pGM-CSF significantly reduced the formation of brain cyst $(*)$ compared to control plasmid-vaccinated mice $(P=0.0014)$. 


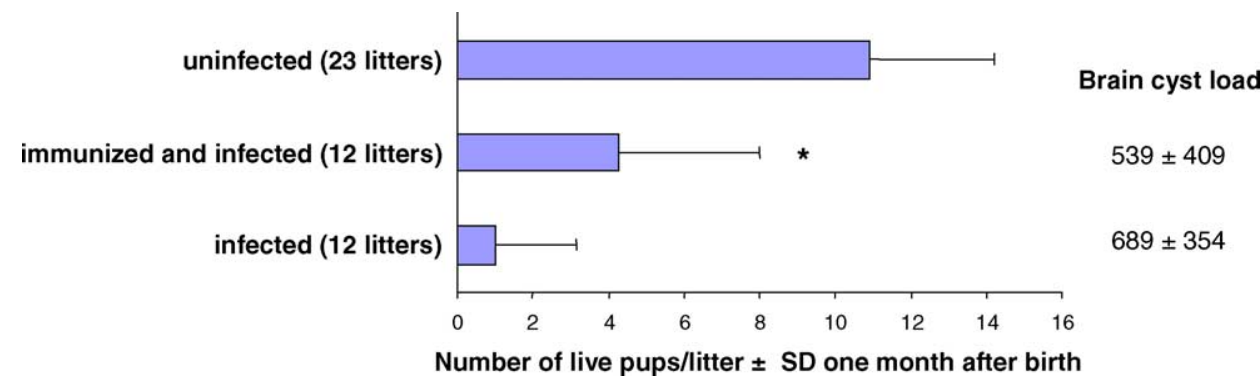

Fig. 4. The effect of vaccination on litters from OF1 mice immunized with pSAG1mut and pGRA4 plus pGM-CSF, prior to infection during gestation with 70 cysts of the 76K strain (between 7th and 10th days of gestation). The mice were immunized on days 0 and 14 with pSAG1mut plus pGRA4 with pGM-CSF (50 $\mu \mathrm{g}$ each). On day 28 , the mice were immunized with plasmids encoding antigens without pGM-CSF. Two control groups were composed of mice left untreated and infected during gestation or left untreated and not infected during gestation. The number of live pups were recorded for 30 days, the values represent the mean \pm S.D. from three independant experiments. ${ }^{*} P=0.05$, immunized and infected group vs. infected group. Brain cyst load was evaluated 30 days after birth.

pGRA4 plus plasmid encoding GM-CSF, exposed to T. gondii in utero, was recorded compared to that from non-immunized mice $(4.25 \pm 3.77$ live pups/litter versus $1.08 \pm 2.15$ live pups/litter, $P<0.05)$. Mortality occured within 5 days after birth. No significant delay in the mortality was observed in the immunized mice but the mortality was lower compared to control mice (Fig. 4). Live pups were weighed when 8-, 15and 30-day-old (Fig. 5). Pups were not weighed before 8 days to avoid risk of cannibalism by the mother because of manipulation of newborns. Eight days after birth, pups born from immunized mice and pups born from uninfected mice did not differ significantly in body weight $(4.49 \pm 0.81 \mathrm{~g}$ versus $4.93 \pm 0.95 \mathrm{~g}$ ) and both groups weighed significantly more than pups born from infected mice $(3.48 \pm 0.65 \mathrm{~g}, P<0.002)$. However, 15 days after birth, pups born from immunized mice significantly weighed less than pups born from uninfected mice $(7.49 \pm 1.46 \mathrm{~g}$ versus $9.65 \pm 1.30 \mathrm{~g}, P<0.0001)$ and the difference in body weight was not quite significant between pups born from immunized mice and pups born from infected mice $(7.49 \pm 1.46 \mathrm{~g}$ versus $6.44 \pm 1.12 \mathrm{~g}, P=0.068)$. The same differences were observed at 30 days after birth.
Pups born from immunized mice significantly weighed less than pups born from uninfected mice $(13.86 \pm 3.20 \mathrm{~g}$ versus $24.71 \pm 2.93 \mathrm{~g}, P<0.0001)$ and the difference in body weight was not quite significant between pups born from immunized mice and pups born from infected mice $(13.86 \pm 3.20 \mathrm{~g}$ versus $11.12 \pm 4.52 \mathrm{~g}, P=0.088)$. The pups were sacrificed 30 days after birth and the brain cyst number was determined. The average number of brain cysts was $689 \pm 354(n=12)$ in pups born from infected mothers and was not statistically different from that of pups born from immunized mothers $(539 \pm 409, n=37)$.

\subsection{Immune responses}

Sera from immunized C57BL/6 mice were collected 2 weeks after the last immunization and analyzed by ELISA for specific anti-SAG1 or anti-GRA4 response. Immunization with pSAG1mut, pGRA4, pSAG1mut plus pGRA4 or pSAG1mut plus pGRA4 and pGM-CSF resulted in a similar and significant antibody production in all the animals whatever the group (Fig. 6).

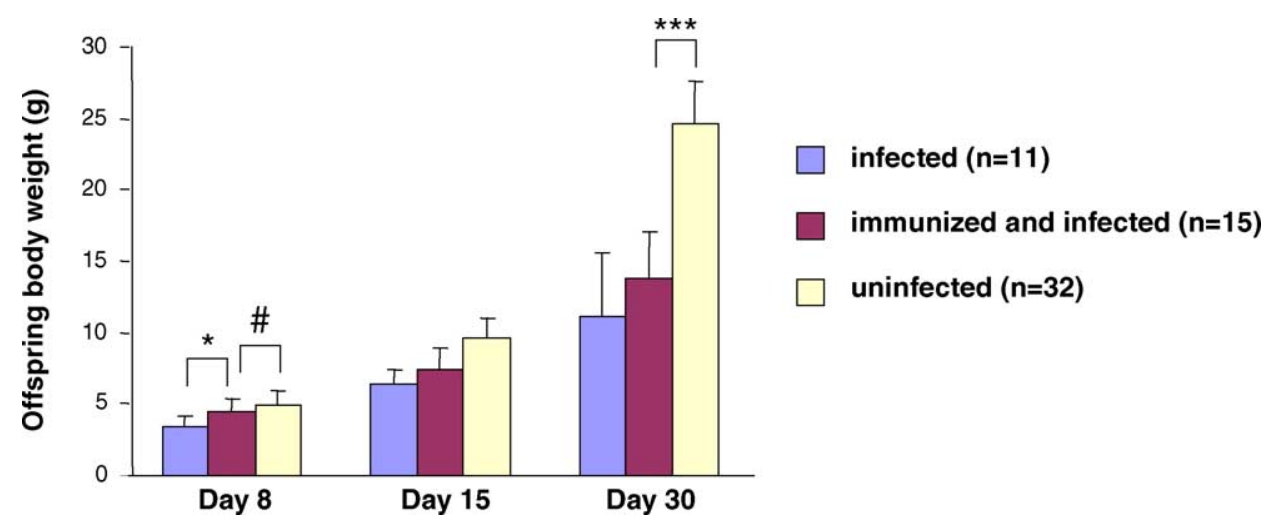

Fig. 5. The effect of vaccination on the body weight of pups exposed to T. gondii in utero. Female OF1 mice were immunized on days 0 and 14 with pSAG1mut plus pGRA4 with pGM-CSF (50 $\mu \mathrm{g}$ each) and were immunized on day 28 with plasmids encoding antigens without pGM-CSF. The mice were infected during pregnancy, between 7th and 10th days of gestation. Two control groups were composed of mice left untreated and infected during gestation or left untreated and not infected during gestation. Pups were weighed on days 8, 15 and 30 following birth. Results from one of three similar experiments are shown and are expressed as the mean \pm S.D. Symbols: ${ }^{*} P<0.002$; ${ }^{* * *} P<0.0001$; (\#) not significant. 

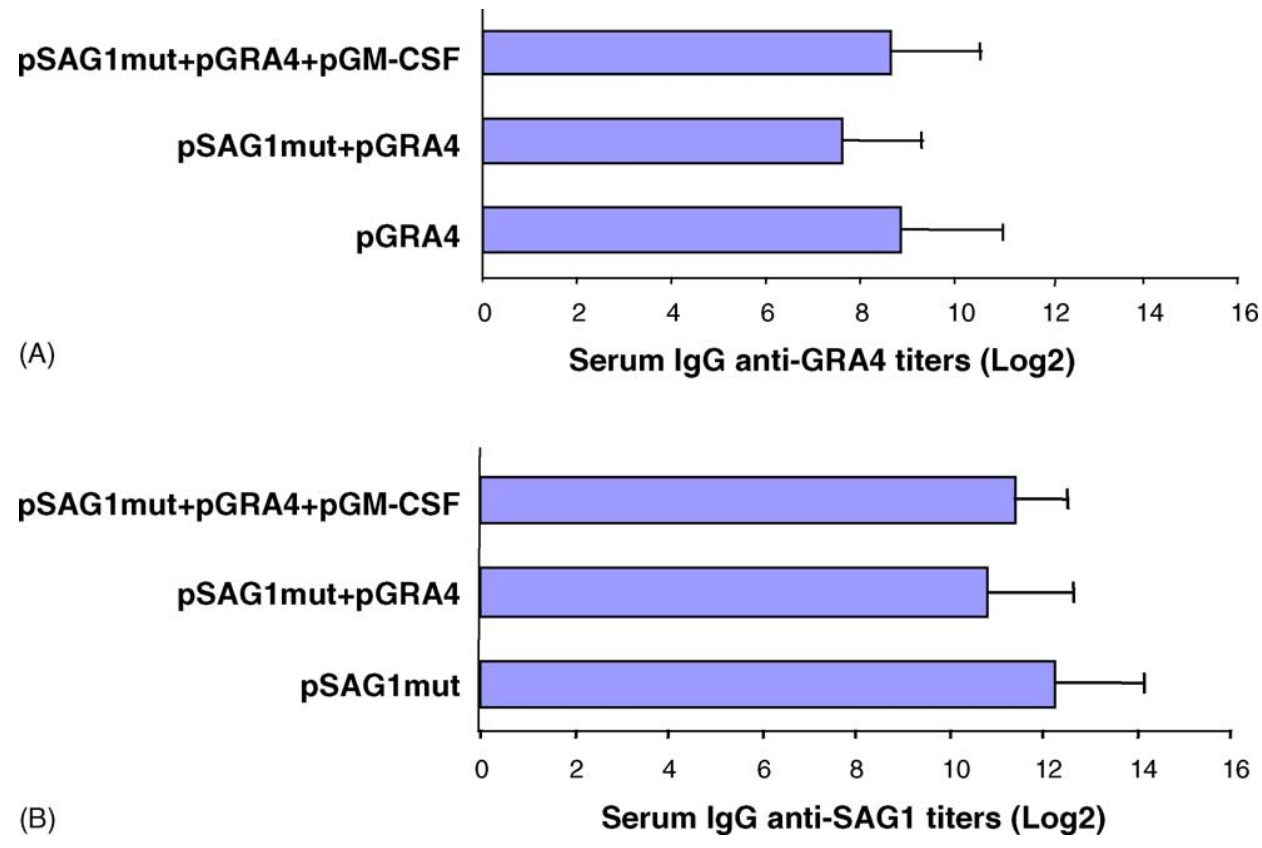

Fig. 6. Serum IgG antibody responses of C57BL/6 mice to GRA4 (A) and to SAG1 (B) following immunization on days 0 and 14 with $100 \mu \mathrm{g}$ pSAG1mut or $100 \mu \mathrm{g}$ pGRA4 or $50 \mu \mathrm{g}$ pSAG1mut plus $50 \mu \mathrm{g}$ pGRA4 or $50 \mu \mathrm{g}$ pSAG1mut plus $50 \mu \mathrm{g}$ pGRA4 with $50 \mu \mathrm{g}$ pGM-CSF. The mice were immunized on day 28 with plasmids encoding antigens without pGM-CSF. Sera were collected on day 43. Titer is given as the reciprocal of the highest dilution whose absorbance was 2.5 fold greater than absorbance of untreated mice sera at the same dilution. Results are expressed as the means $\log 2$ titers \pm S.D. These data are representative of two independent experiments.

To evaluate cellular anti-Toxoplasma immune responses in the DNA-vaccinated C57BL/6 mice, spleen cells were prepared 6 days after the last immunization and were stimulated in vitro with $T$. gondii RH TAg (Table 1). Splenocytes from mice immunized with pGRA4 alone showed a slight proliferative response $(P=0.0003$ versus control pcDNA3). The proliferative response of splenocytes from mice immunized with pSAG1mut alone was not quite significative $(P=0.0546$ versus control pcDNA3). A substantial specific proliferative response was observed in spleen cell cultures from mice immunized with pSAG1mut plus pGRA4 $(P=0.03$ versus
pGRA4) and this response was markedly enhanced by pGMCSF co-administration $(P=0.002$ versus pSAG1mut plus pGRA4). In addition, spleen cells from all immunized and control groups proliferated to comparable levels in response to the mitogen, Con A (IS > 10, data not shown). Spleen cells from mice immunized with pSAG1mut, pSAG1mut plus pGRA4 or pSAG1mut plus pGRA4 and pGM-CSF produced greater levels of IFN- $\gamma$ when stimulated with TAg (133 pg/ml for $\mathrm{pSAG} 1 \mathrm{mut}, 392 \mathrm{pg} / \mathrm{ml}$ for $\mathrm{pSAG} 1 \mathrm{mut}$ plus pGRA4, $1197 \mathrm{pg} / \mathrm{ml}$ for pSAG1mut plus pGRA4 and pGM$\mathrm{CSF}$ ) than splenocytes from mice inoculated with pcDNA3

Table 1

Cellular immune responses of spleen cells from C57BL/6 mice immunized i.m. with pSAG1mut, pGRA4, pSAG1mut plus pGRA4 or pSAG1mut plus pGRA4 with pGM-CSF

\begin{tabular}{|c|c|c|c|c|}
\hline \multirow[t]{2}{*}{ Immunization regimen $^{\mathrm{a}}$} & \multirow[t]{2}{*}{ Proliferation $\mathrm{SI}^{\mathrm{b}}$} & \multicolumn{3}{|c|}{ Cytokine production $(\mathrm{pg} / \mathrm{ml})^{\mathrm{c}}$} \\
\hline & & IFN- $\gamma$ & IL-4 & IL-10 \\
\hline Control pcDNA3 & $1.33 \pm 0.34$ & $<20^{\mathrm{d}}$ & $<20^{\mathrm{d}}$ & $<20^{\mathrm{d}}$ \\
\hline pSAG1mut & $2.7 \pm 0.8$ & $133 \pm 63$ & $<20^{\mathrm{d}}$ & $<20^{\mathrm{d}}$ \\
\hline pGRA4 & $4.11 \pm 0.2$ & $26 \pm 5$ & $<20^{\mathrm{d}}$ & $<20^{\mathrm{d}}$ \\
\hline pSAG1mut + pGRA4 & $4.86 \pm 0.35$ & $392 \pm 50$ & $<20^{\mathrm{d}}$ & $<20^{\mathrm{d}}$ \\
\hline pSAG1mut + pGRA4 + pGM-CSF & $17.29 \pm 1.96$ & $1197 \pm 50^{* * * *}$ & $<20^{\mathrm{d}}$ & $<20^{\mathrm{d}}$ \\
\hline
\end{tabular}

${ }^{\text {a }}$ Mice were immunized on days 0 and 14 with $100 \mu \mathrm{g}$ pSAG1mut or $100 \mu \mathrm{g}$ pGRA4 or $50 \mu \mathrm{g}$ pSAG1mut plus $50 \mu \mathrm{g}$ pGRA4 or $50 \mu \mathrm{g}$ pSAG1mut plus $50 \mu \mathrm{g}$ pGRA4 with $50 \mu \mathrm{g}$ pGM-CSF. The mice were immunized on day 28 with plasmids encoding antigens without pGM-CSF. Splenocytes from 3 mice per experimental group were recovered 6 days after the last immunization and cultured with $15 \mu \mathrm{g} / \mathrm{ml} \mathrm{TAg}$.

${ }^{b}$ SI: stimulation index, expressed as the ratio between the counts per minute of stimulated and unstimulated cells.

${ }^{c}$ The culture supernatants were examined for cytokine production. Values for IFN- $\gamma$, IL-4 and IL-10 were obtained from $96 \mathrm{~h}, 24 \mathrm{~h}$ and $72 \mathrm{~h}$ culture, respectively.

${ }^{\mathrm{d}}$ Below threshold of assay sensitivity.

*** $P<0.0001$ pSAG1mut + pGRA4 + pGM-CSF vs. pSAG1 + pGRA4. 
Table 2

Cellular immune responses of spleen cells from OF1 mice immunized i.m. with pSAG1mut plus pGRA4 with pGM-CSF

\begin{tabular}{|c|c|c|c|c|}
\hline \multirow[t]{2}{*}{ Immunization regimen ${ }^{\mathrm{a}}$} & \multirow[t]{2}{*}{ Proliferation $\mathrm{SI}^{\mathrm{b}}$} & \multicolumn{3}{|c|}{ Cytokine production $(\mathrm{pg} / \mathrm{ml})^{\mathrm{c}}$} \\
\hline & & IFN- $\gamma$ & IL-4 & IL-10 \\
\hline Untreated & $1.1 \pm 0.05$ & $4470 \pm 3440$ & $<20^{\mathrm{d}}$ & $<50^{\mathrm{d}}$ \\
\hline Control pcDNA3 & $1.12 \pm 0.06$ & $510 \pm 10$ & $<20^{\mathrm{d}}$ & $<50^{\mathrm{d}}$ \\
\hline pSAG1mut + pGRA4 + pGM-CSF & $5.49 \pm 0.42^{* *}$ & $25,000 \pm 4580^{*}$ & $<20^{\mathrm{d}}$ & $535 \pm 527$ \\
\hline \multicolumn{5}{|c|}{$\begin{array}{l}\text { a Mice were immunized on days } 0 \text { and } 14 \text { with } 50 \mu \mathrm{g} \text { pSAG1mut plus } 50 \mu \mathrm{g} \text { pGRA4 with } 50 \mu \mathrm{g} \text { pM-CSF and were immunized on day } 28 \text { with plasmids } \\
\text { encoding antigens without pGM-CSF. Splenocytes from } 4 \text { mice per experimental group were recovered } 6 \text { days after the last immunization and cultured with } \\
10 \mu \mathrm{g} / \mathrm{ml} \text { TAg. }\end{array}$} \\
\hline $10 \mu \mathrm{g} / \mathrm{ml} \mathrm{TAg}$ & \multicolumn{4}{|c|}{ b SI: stimulation index, expressed as the ratio between the counts per minute of stimulated and unstimulated cells. } \\
\hline \multirow{2}{*}{\multicolumn{5}{|c|}{$\begin{array}{l}\mathrm{c} \text { The culture supernatants were examined for cytokine production. Values for IFN- } \gamma \text {, IL- } 4 \text { and IL-10 were obtained from } 96 \mathrm{~h}, 24 \mathrm{~h} \text { and } 72 \mathrm{~h} \text { culture, } \\
\text { respectively. }\end{array}$}} \\
\hline & & & & \\
\hline \multicolumn{5}{|c|}{${ }^{\mathrm{d}}$ Below threshold of assay sensitivity. } \\
\hline \multicolumn{5}{|c|}{$* P=0.0015$, pSAG1mut + pGRA4 + pGM-CSF vs. untreated. } \\
\hline \multicolumn{5}{|c|}{ ** $P=0.0042$, pSAG1mut + pGRA4 + pGM-CSF vs. pcDNA3. } \\
\hline
\end{tabular}

(not detected, $<20 \mathrm{pg} / \mathrm{ml}$ ) (Table 1). Splenocytes from mice immunized with pGRA4 produced low amounts of IFN- $\gamma$ $(26 \mathrm{pg} / \mathrm{ml})$. Co-administration of pGM-CSF with pSAG1mut plus pGRA4 enhanced IFN- $\gamma$ production $(P<0.0001$ versus pSAG1mut plus pGRA4). We investigated whether splenocytes from immunized mice secreted Th2-associated cytokines. IL-10 and IL-4 were not detected in the cultures from any groups.

Vaccination of Swiss OF1 mice with a mixture of pSAG1mut, pGRA4 plus plasmid encoding GM-CSF induced an antibody response against SAG1 in $100 \%$ of the immunized mice and an antibody response against GRA4 in about $50 \%$ of the immunized mice. Whatever the proteins, antibody production observed in OF1 mice was lower than that observed in C57BL/6 mice (anti-SAG1 IgG titers: $7.5 \pm 1.2$, anti-GRA4 IgG titers: $7.1 \pm 0.8$ ). In order to characterize whether a Th1 and/or Th2 response was elicited in immunized mice, the distribution of IgG sub-types was analyzed against $T$. gondii RH TAg (Fig. 7). A predominance of anti-T. gondii $\operatorname{IgG} 2$ a over IgG1 was observed in sera from

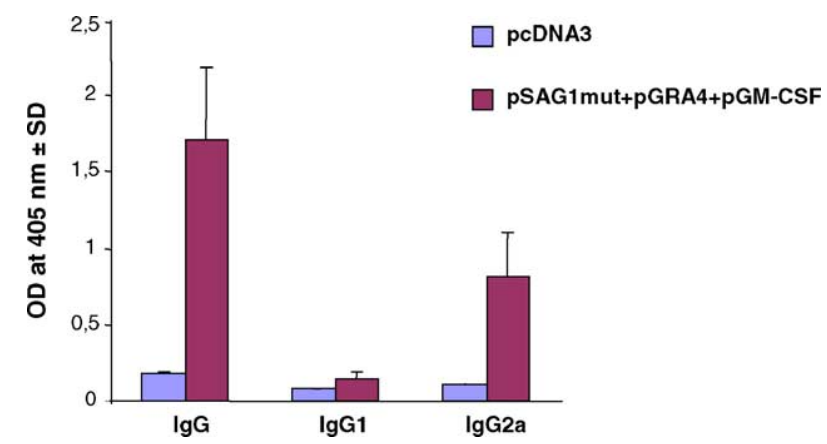

Fig. 7. Determination of the specific anti-T. gondii IgG subclass profile in the sera of OF1 mice immunized with pSAG1mut and pGRA4 plus pGMCSF. Female OF1 mice were immunized on days 0 and 14 with pSAG1mut plus pGRA4 with pGM-CSF ( $50 \mu \mathrm{g}$ each) and were immunized on day 28 with plasmids encoding antigens without pGM-CSF. Sera were collected on day 43 and analyzed by ELISA using TAg. The results are expressed as the mean of the $\mathrm{OD}_{405} \pm$ S.D. and represent one of three similar experiments. immunized mice implying that vaccination with pSAG1mut, pGRA4, plus pGM-CSF elicits a Th1 humoral response.

To evaluate cellular anti-Toxoplasma immune responses, spleen cell suspensions from immunized Swiss OF1 mice were stimulated in vitro with $T$. gondii RH TAg (Table 2). A specific proliferative response was observed in spleen cell cultures from vaccinated mice. In addition, spleen cells from all immunized and control mice proliferated to comparable levels in response to the mitogen, Con A (IS > 10, data not shown). Spleen cells from vaccinated mice produced significant levels of IFN- $\gamma$ when stimulated with TAg $(25 \mathrm{ng} / \mathrm{ml}$, $P=0.0015$ versus untreated control). IL- 4 was undetectable in splenocytes supernatants from controls (untreated and pcDNA3) and vaccinated mice. Il-10 was produced by restimulated splenocytes from immunized mice $(535 \mathrm{pg} / \mathrm{ml})$ and no specific release was demonstrated in splenocytes supernatants from controls (untreated and pcDNA3). These results suggested a modulated Th1 response.

\section{Discussion}

Our data demonstrated that a multiantigenic DNA-vaccine using pSAG1mut and pGRA4 with a plasmid encoding GMCSF was able to mount a significant protection against acute and chronical infection and a partial protection against congenital toxoplasmosis.

In the present study, the DNA vaccine vector based on the SAG1 gene contains the natural signal sequence of SAG1. The results from pSAG1-transfected COS cells showed that the recombinant SAG1 is expressed in an immunologically active form which can be recognized under non-reducing conditions by antibodies from infected mice. The recombinant protein is bound to the cell membrane (data not shown) and is $\mathrm{N}$-glycosylated unlike the native SAG1. Others have shown that the glycosylation of the expressed SAG1 has little influence on the immunoreactivity of the molecule [10]. Mutation on the $\mathrm{N}$-glycosylation site (the Asn 241 was substituted for Gln codon by point mutation) resulted in the expression of an 
immunologically active protein with an apparent molecular weight indistinguishable from that of the protein found in the parasite. This latter form was used in these studies.

Immunization of C57BL/6 mice with a mixture of plasmids expressing SAG1 and GRA4 resulted in an improvement of the protective immunity against the acute phase of a $76 \mathrm{~K}$-parasite infection as measured by the survival rate, in comparison with mice immunized with plasmid expressing GRA4 alone or SAG1 alone. These results suggested that DNA cocktail immunization might be an important approach to achieve an effective vaccine against $T$. gondii. Our findings are consistent with the recent results showing that coinjection of low dose of two genes encoding SAG1 and ROP2 afforded protection while single plasmid have no protective effect [25]. Several studies have reported that the magnitude of the immune response to a DNA vaccine can be enhanced by injecting a plasmid encoding mouse GM-CSF [26]. GMCSF facilitates the induction of primary immune responses by activating and recruiting antigen-presenting cells [27]. The effect of immunization with plasmids expressing GRA4 and SAG1 co-inoculated with a plasmid encoding GM-CSF was therefore investigated as it was done in previous studies for SAG1 [10] or GRA4 [12]. This immunization improved the protective immunity compared to a mixture of plasmids expressing SAG1 and GRA4. In addition to determine whether it is possible to protect against mortality from acute toxoplasmosis, we were interested in determining whether vaccination would provide any protection against the formation of T. gondii tissue cysts in the brain and also against congenital toxoplasmosis. For these experiments outbred Swiss OF1 mice were vaccinated with a mixture of plasmids expressing SAG1 and GRA4 plus plasmid encoding GM-CSF. These mice were chosen to circumvent genetic restriction and because they are intermediatly resistant. Although the vaccination did not completely eliminate tissue cysts in the animals, a substantial reduction in the cyst burden was observed in comparison to control animals.

It is well established that both humoral and cell-mediated immune responses are important in conferring immunity to T. gondii infection [28,29]. Cellular protective immunity is known to be mediated mainly by $\mathrm{T}$ lymphocytes and gamma interferon has been shown to be the main cytokine involved in protection [30]. DNA vaccination with a plasmid encoding SAG1 or a plasmid encoding GRA4 induced partial protection in C57BL/6 mice and the responses were assiociated with a Th1-type profile $[10,12]$. We confirmed here that DNA vaccination of C57BL/6 mice with a plasmid encoding SAG1 or GRA4 induced secretion of IFN- $\gamma$. However, in our experimental conditions, cells from mice vaccinated with the plasmid encoding SAG1 did not significantly proliferate when stimulated with TAg. Furthermore, co-inoculation of a plasmid encoding SAG1 with a plamid encoding GRA4 potentiates these reponses and the association of a plasmid encoding GM-CSF greatly increases the proliferative response and IFN- $\gamma$ production. Swiss OF1 mice vaccinated with a mixture of pSAG1mut, pGRA4 plus plasmid encoding
GM-CSF also showed specific cellular immune responses characterized by significantly increased splenocyte proliferation and secretion of IFN- $\gamma$ in response to TAg. As already described, DNA vaccination of $\mathrm{C} 57 \mathrm{BL} / 6$ mice with a plasmid encoding GRA4 or SAG1 induces specific humoral responses. The levels of anti-SAG1 and anti-GRA4 in OF1 mice were constantly lower than those in C57BL/6 mice. The heterogeneity of the immunes responses in the Swiss OF1 mice could result from the outbred character of these mice. Nevertheless all responder mice tested showed an humoral type- 1 response and a cellular type-1 response.

In an attempt to evaluate protection against congenital toxoplasmosis, we performed preliminary experiments with OF1 mice to demonstrate that OF1 mice can be used as a model of congenital toxoplasmosis. Infection of pregnant mice between the 7 th and 10th days of pregnancy with 70 cysts of the $76 \mathrm{~K}$ strain by the oral route results in a high degree of mortality following birth. Surviving pups displayed body weights strongly reduced as compared to those from uninfected mice. The infection status of the offspring determined by brain cyst load at 30 days after birth, showed that all stillborn pups are infected. In this model, we tested the protective effect of a chronic infection when mice were reinfected during pregnancy by the same $76 \mathrm{~K}$ strain. All pups survived, they did not weight less than uninfected controls at weaning age, furthermore, they exhibited significantly fewer cysts in the brain than infected controls ( $90 \%$ cysts reduction). Tachyzoites have been found in the milk of several species, including sheep, goats, cows and mice [23,24]. In order to prevent the possibility of infection through lactation others fostered the litters to uninfected lactating dams [11,31,32]. Without fostering litters to uninfected lactating mice we observed a strong protection. In conclusion, even if some mice may become infected via maternal milk, OF1 dams develop immunity capable of protecting their pups even if reinfection occurs during pregnancy. Ideally, a sucessful vaccine should induce a similar level of protection. Importantly, the survival rates obtained in litters from mice immunized with a mixture of pSAG1mut, pGRA4 plus plasmid encoding GM-CSF were significantly higher than those obtained from infected controls and 8 days after birth, pups born from immunized mice did not weight less than pups born from uninfected mice. However, pups born from immunized mothers weighed less than pups born from uninfected mothers at days 15 and 30 after birth and exhibited numerous brains cysts 30 days after birth. It is of note that one pregnant immunized mouse was sacrificed on day 9 post infection, the PCR results, targeting the B1 gene carried out on fetuses, suggest in utero infection (data not shown). In our experimental conditions, the partial protection may be attributed to a delay and or to a less degree of foetus infection. Conflicting results have been reported about the protective effect of SAG1 against maternofoetal transmission using, BALB/c mice, pups fostered or pregnant females sacrificed on the last day of gestation and orally challenged with type II strain (Me49 or Beverly) [7,11]. Although the immune response generated by DNA vaccination with 
SAG1 was clearly of type-1 nature and appropriate for protection against adult acquired $T$. gondii infection following oral challenge with cysts of the Beverly type II strain as measured by mortality or brain cyst burden, vaccination of dams did not prevent maternofoetal transmission [11]. However, immunization of BALB/c mice with a recombinant SAG1 protein reduced the number of infected fetuses and was associated with a mixed type- 1 and type- 2 immunity following oral challenge with cysts of the Me49 type II strain of $T$. gondii [7]. The ability of SAG1 to prevent congenital infection was also evaluated in a guinea pig model [33]. Subcutaneous immunization of guinea pigs with the recombinant SAG1 protein expressed in Pichia pastoris, formulated with SBAS1, a Th1 response inducer adjuvant, elicits significant protection against maternofetal transmission following challenge with tachyzoites during pregnancy.

Pregnant mice are more susceptible to infection than nonpregnant mice $[34,35]$. Susceptibility of the pregnant host to T. gondii infection may be due to a type- 2 cytokine pattern maintained during gestation $[35,36]$. The type- 2 cytokine bias has been characterized in the murine placenta and is associated with successful implantation, maintenance of early pregnancy, and supression of local inflammatory [37,38]. Shirahata et al. [35] have reported that the depressed production of IFN- $\gamma$ appears to be correlated with the increased susceptibility to primary $T$. gondii infection in pregnant mice and that administration of recombinant murine IFN- $\gamma$ enhances host resistance of pregnant mice. Furthermore, administration of recombinant IL-2 enhances host resistance of pregnant mice to a lethal challenge with $T$. gondii and this administration resulted in the augmented production of both IFN- $\alpha$ and IFN- $\gamma$ in pregnant mice [39]. But recently, Abou-Bacar et al. [40], showed that neutralization of IFN- $\gamma$ in orally infected BALB/c mice on day 11 of gestation with cysts of the PRU type II strain, decreased congenital $T$. gondii transmission, contrasting with an exacerbation of maternal infection. The role of IL-4 in the regulation of congenital toxoplasmosis is controversial. Naive IL-4 knockout B6/129 pregnant mice were shown more resistant to T. gondii infection when infected during gestation. However, they failed to protect their fetuses from infection [41]. Others have shown that $T$. gondii congenital transmission was decreased by as much as $50 \%$ in IL-4 deficient BALB/c mice [36]. These investigations focused on innate immunity. Long and Baszler [42] have compared induction of innate and adaptative immune responses against protozoal congenital transmission. Neutralisation of maternal IL-4 during gestation does little change the frequency of congenital Neospora caninum transmission whereas manipulation of antigen specific maternal immune response before gestation, specially down regulation of type-2 antigen specific immune responses before gestation results in significant decrease of congenital transmission after challenge infection during gestation.

DNA-based vaccination has been used successfully to elicit both humoral and type-1 cellular immune responses and has been particularly useful in inducing cytotoxic T cells. Im- munization of BALB/c mice with a plasmid encoding SAG1 did not confer protection against congenital toxoplasmosis following oral infection with cysts of the type II Beverly strain on day 11 of gestation [11]. However, Roberts et al. [31] using BALB/c mice and oral infection with the same $T$. gondii strain demonstrated that soluble tachyzoite antigens (STAg). entrapped within non-ionic surfactant (NISV) significantly confered protection against fetal death and vertical transmission. STAg emulsified in FCA or without adjuvant did not confer protection. Only splenocytes from mice immunized with STAg entrapped in NISVproduced IFN- $\gamma$ which levels were significantly different from those produced by splenocytes from non-vaccinated mice in presence of TAg. These results clearly suggested that a type- 1 response is protective and that a multivalent vaccine would be more effective in this disease model.

So, it is evident that complete immunity against toxoplasmosis can not be elicited by a single $T$. gondii protein. Therefore the results presented here demonstrated that GRA4 and SAG1 should be components in the development of a multiantigenic vaccine against toxoplasmosis.

\section{Acknowledgements}

The authors would like to thank J. Pierre, J.M. Rith, T. Papin and D. Tabareau for their excellent technical assistance, Jean-François Dubremetz for the gift of the Mab 1E5 and H.C.J. Ertl for the gift of GM-CSF plasmid.

\section{References}

[1] Buxton D, Thomson K, Maley S, Wright S, Bos HJ. Vaccination of sheep with a live incomplete strain (S48) of Toxoplasma gondii and their immunity to challenge when pregnant. Vet Rec 1991;129(5):89-93.

[2] Khan IA, Ely KH, Kasper LH. A purified parasite antigen (P30) mediates CD8+ Tcells immunity against fatal Toxoplasma gondii infection in mice. J Immunol 1991;147:3501-6.

[3] Debard N, Buzoni-Gatel D, Bout D. Intranasal immunization with SAG1 protein of Toxoplasma gondii in association with cholera toxin dramatically reduces development of cerebral cysts after oral infection. Infect Immun 1996;64(6):2158-66.

[4] Darcy F, Maes P, Grass-Masse H, Auriault C, Bossus M, Deslee $\mathrm{D}$, et al. Protection of mice and nude rats against toxoplasmosis by a multiple antigenic peptide construction derived from Toxoplasma gondii P30 antigen. J Immunol 1992;14:3636-41.

[5] Letscher-Bru V, Villard O, Risse B, Zauke M, Klein J-P, Kein TT. Protective effect of vaccination with a combination of recombinant surface antigen 1 and interleukine-12 against toxoplasmosis in mice. Infect Immun 1998;66:4503-6.

[6] Petersen E, Nielsen HV, Christiansen L, Spenter J. Immunization with $E$. coli produced recombinant $T$. gondii SAG1 with alum as adjuvant protect mice against lethal infection with Toxoplasma gondii. Vaccine 1998;16:1283-9.

[7] Letscher-Bru V, Pfaff AW, Abou-Bacar A, Filisetti D, Antoni E, Villard $\mathrm{O}$, et al. Vaccination with Toxoplasma gondii SAG-1 protein is protective against congenital toxoplasmosis in BALB/c mice but not in CBA/J mice. Infect Immun 2003;71(11):6615-9. 
[8] Pertmer TM, Roberts TR, Haynes JR. Influenza virus nucleoproteinspecific immunoglobulin $\mathrm{G}$ subclass and cytokine responses elicited by DNA vaccination are dependent on the route of vector DNA delivery. J Virol 1996;70:6119-25.

[9] Nielsen HV, Lauemoller SL, Christiansen L, Buus S, Fomsgaard A, Petersen E. Complete protection against lethal Toxoplasma gondii infection in mice immunized with a plasmid encoding the SAG1 gene. Infect Immun 1999;67(12):6358-63.

[10] Angus CW, Klivington-Evans D, Dubey JP, Kovacs JA. Immunization with a DNA plasmid encoding the SAG1 (P30) protein of Toxoplasma gondii is immunogenic and protective in rodents. J Infect Dis 2000;181(1):317-24.

[11] Couper KN, Nielsen HV, Petersen E, Roberts F, Roberts CW, Alexander J. DNA vaccination with the immunodominant tachyzoite surface antigen (SAG-1) protects against adult acquired Toxoplasma gondii infection but does not prevent maternofoetal transmission. Vaccine 2003;21:2813-20

[12] Desolme B, Mévélec M-N, Buzoni-Gatel D, Bout D. Induction of protective immunity against toxoplasmosis in mice by DNA immunization with a plasmid encoding Toxoplasma gondii GRA4 gene. Vaccine 2000;18(23):2512-21.

[13] Ivory C, Chadee K. DNA vaccines: designing strategies against parasitic infections. Genet Vaccines Ther 2004;2(1):17-25.

[14] Laugier M, Quilici M. Intérêt expérimental d'une souche de toxoplasme peu pathogene pour la souris. Ann Parasitol Hum Comp 1970;45:389-403.

[15] Ajzenberg D, Banuls AL, Tibayrenc M, Darde ML. Microsatellite analysis of Toxoplasma gondii shows considerable polymorphism srtuctured into two main groups. Int J Parasitol 2002;32(1):27-38.

[16] Ajzenberg D, Cogne N, Paris L, Bessieres MH, Thulliez P, Filisetti $\mathrm{P}$, et al. Genotype of 86 Toxoplasma gondii isolates associated with human congenital toxoplasmosis, and correlation with clinical findings. J Infect Dis 2002;186(5):684-9.

[17] Burg JL, Perelman D, Kasper LH, Ware PL, Boothroyd JC. Molecular analysis of the gene encoding the major surface antigen of Toxoplasma gondii. J Immunol 1988;141:3584-91.

[18] Chardès T, Bourguin I, Mévélec M-N, Dubremetz JF, Bout D. Antibody responses to Toxoplasma gondii in sera, intestinal secretions, and milk from orally infected mice and characterization of target antigens. Infect Immun 1990;58(5):1240-6.

[19] Couvreur G, Sadak A, Fortier B, Dubremetz JF. Surface antigens of Toxoplasma gondii. Parasitol 1988;97:1-10.

[20] Davis HL, Whalen RG, Demeneix BA. Direct gene transfer into skeletal muscle in vivo: factors affecting efficiency of transfer and stability of expression. Hum Gene Ther 1993;4:151-9.

[21] Mévélec M-N, Mercereau-Puijalon O, Buzoni Gatel D, Bourguin I, Chardès T, Dubremetz JF, et al. Mapping of B epitopes in GRA4, a dense granule antigen of Toxoplasma gondii and protection studies using recombinant proteins administered by oral route. Parasite Immunol 1998;20:183-95.

[22] Sharma SD, Mullenak J, Araujo FG, Erlich HA, Remington JS Western blot analysis of the antigens of Toxoplasma gondii recognized by human IgM and IgG antibodies. J Immunol 1983;131: 977-83.

[23] Pettersen EK. Transmission of toxoplasmosis via milk from lactating mice. Acta Pathol Microbiol Immunol Scand 1984;92(3):175-6.

[24] Tenter AM, Heckeroth A, Weiss LM. Toxoplasma gondii: from animals to humans. Int J Parasitol 2000;30:1217-58.

[25] Fachado A, Rodriguez A, Angel SO, Pinta AC, Vila L, Acosta A, et al. Protective effect of a naked DNA vaccine cocktail against lethal toxoplasmosis in mice. Vaccine 2003;21:1327-35.
[26] Warren TL, Weiner GJ. Uses of granulocyte-macrophage colonystimulating factor in vaccine development. Curr Opin Hematol 2000;7:168-73.

[27] Kim JJ, Yang JS, Lee DJ, Wilson DM, Nottingham LK, Morrison $\mathrm{L}$, et al. Macrophage colony-stimulating factor can modulate immune responses and attract dendritic cells in vivo. Hum Gene Ther 2000;11:305-21.

[28] Kang H, Remington JS, Suzuki Y. Decreased resistance of B cell deficient mice to infection with $T$. gondii despite unimpaired expression of IFN-gamma, TNF-alfa and inducible nitric oxide synthasem. J Immunol 2000;164:2629-34.

[29] Gazzinelli RT, Hakim FT, Hieny S, Shearer GM, Sher A. Synergistic role of CD4+ and CD8+ T lymphocytes in IFN-gamma production and protective immunity induced by an attenuated Toxoplasma gondii vaccine. J Immunol 1991;146:286-92.

[30] Suzuki Y, Orellana MA, Shreiber RD, Remington JS. Interferon- $\gamma$ : the major mediator of resistance against Toxoplasma gondii. Science 1988;240:516-8.

[31] Roberts CW, Brewer JM, Alexander J. Congenital toxoplasmosis in the Balb/c mouse: prevention of vertical disease transmission and fetal death by vaccination. Vaccine 1994;12(15):138994.

[32] Elsaid MMA, Martins MS, Frézard F, Braga EM, Vitor RWA. Vertical toxoplasmosis in a murine model. Protection after immunization with antigens of Toxoplasma gondii incorporated into liposomes. Mem Inst Oswaldo Cruz 2001;96(1):99-104.

[33] Haumont M, Delhaye L, Garcia L, et al. Protective immunity against congenital toxoplasmosis with recombinant SAG1 protein in a guinea pig model. Infect Immun 2000;68(9):4948-53.

[34] Luft BJ, Remington JS. Effect of pregnancy on resistance to Listeria monocytogenes and Toxoplasma gondii infections in mice. Infect Immun 1982;38:1164-71.

[35] Shirahata T, Muroya N, Ohta C, Goto H, Nakane A. Correlation between increased susceptibility to primary Toxoplasma gondii infection and depressed production of gamma interferon in pregant mice. Microbiol Immunol 1992;36(1):81-91.

[36] Thouvenin M, Candolfi E, Villard O, Klein JP, Kien T. Immune response in a murine model of congenital toxoplasmosis: increased susceptibility of pregnant mice and transplacental passage of Toxoplasma gondii are type 2-dependant. Parasitologia 1997;39(4):279-83.

[37] Lin H, Mosmann TR, Guilbert L, Tuntipopipat S, Wegmann TG. Synthesis of T helper 2-type cytokines at the maternal-fetal interface. J Immunol 1993;151:4562.

[38] Delassus S, Coutinho GC, Saucier C, Darche S, Kourilsky P. Differential cytokine expression in maternal blood and placenta during murine gestation. J Immunol 1994;152:2411.

[39] Shirahata T, Muroya N, Ohta C, Goto H, Nakane A. Enhancement by recombinant human interleukin 2 of host resistance to Toxoplasma gondii infection in pregnant mice. Microbiol Immunol 1993;37(7):583-90.

[40] Abou-Bacar A, Pfaff AW, Georges S, Letscher-Bru V, Filisetti D, Villard O, et al. Role of NK cells and gamma interferon in transplacental passage of Toxoplasma gondii in a mouse model of primary infection. Infect Immun 2004;72(3):1397-401.

[41] Alexander J, Jebbari H, Bluethmann H, Brombacher F, Roberts CW. The role of IL-4 in adult acquired and congenital toxoplasmosis. Int J Parasitol 1998;28:113-20.

[42] Long MT, Baszler TV. Neutralization of maternal IL-4 modulates congenital protozoal transmission: comparison of innate versus acquired immune responses. J Immunol 2000;164:4768-74. 